\title{
Hémoglobine recombinante et transporteur d'oxygène artificiel
}

Les drames récents de la transfusion sanguine ont stimulé les recherches destinées à remplacer le sang par des solutés synthétiques permettant $d$ 'assurer une bonne fonction du transport et de la délivrance de l'oxygène. Des progrès récents ont permis d'aboutir à des hémoglobines recombinantes $d$ 'affinité diminuée pour l'oxygène (ce qui permet une meilleure oxygénation des tissus) et dont la structure tétramérique est stabilisée par une liaison covalente, évitant ainsi la dissociation en dimères néphrotoxiques. Reste à éviter l'oxydation rapide de cette molécule en méthémoglobine inactive et à en améliorer la production. La création de gros animaux transgéniques - déjà entreprise - pourrait ici constituer une solution d'avenir.

\section{Josée Pagnier Claude Poyart}

Remerciements

Les travaux effectués dans notre unité ont été financés par la société l'Air liquide, la direction des Recherches, Études et Techniques, (contrat $\left.n^{\circ} 89 / 245\right)$, l'institut Mérieux, et l'Inserm.

\section{ADRESSE}

J. Pagnier: docteur ès sciences, directeur de recher che à l'Inserm. C. Poyart : docteur en médecine, directeur de recherche à l'Inserm. Inserm U. 299 hôpital de Bicêtre, 94275 Le Kremlin-

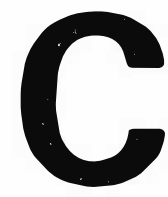

hez les mammifères, l'hémoglobine $(\mathrm{Hb})$ assure le transport des gaz respiratoires, oxygène et gaz carbonique. Jusqu'à présent, le seul moyen efficace de compenser une perte importante de sang a été et reste la transfusion sanguine devenue, au moins dans les pays économiquement développés, un acte routinier mais loin d'être anodin. Les problèmes qui lui sont liés - risques infectieux et immunologiques notamment - ont depuis plusieurs décennies, conduit à rechercher un transporteur d'oxygène de remplacement. Les virus du SIDA, des hépatites B et C, la crainte de voir apparaître de nouveaux vecteurs de maladies encore inconnues sont autant de facteurs qui rendent impérative et urgente la mise au point d'un substitut à la transfusion sanguine. Les deux approches les plus étudiées concernent : l'une, des produits de synthèse chimique, les perfluorocarbones (PFC) ; l'autre, les solutions d'Hb [1-3].

Les PFC sont d'excellents solvants de l'oxygène. Émulsionnés dans le plasma, ils augmentent la quantité d'oxygène dissous qui est directement proportionnelle à la pression partielle en oxygène (loi de Henry) mais reste cependant faible comparée à la quantité d'oxygène combinée à l'hémoglobine. Les PFC n'atteignent leur maximum d'efficacité qu'en atmosphère très enrichie en oxygène. Leur insolubilité en milieu aqueux et leur instabilité imposent une préparation préalable à leur injection. Ces inconvénients limitent leur utilisation en urgence, sur le terrain.

L'utilisation de solutions d'Hb, stratégie plus ancienne, "physiologique ", $m / s n^{\circ} 8$, vol. 8, octobre 92 
consiste à modifier la molécule d' $\mathrm{Hb}$ (humaine ou bovine) par voie chimique, afin de la rendre apte, en solution, au transport de l'oxygène in vivo $[2,3]$. Le développement des biotechnologies peut contribuer à la réalisation de cet objectif ambitieux qui implique la maîtrise d'une somme importante de connaissances sur l' $\mathrm{Hb}$ [4-6]. Modifier la structure de l'Hb par ingénierie génétique ne saurait être réalisé au hasard. Les stratégies possibles peuvent être suggérées par l'analyse des nombreuses $\mathrm{Hb}$ mutées naturelles [7] et la connaissance de la structure cristallographique pour certaines d'entre elles [8]. D'autres informations proviennent de la biochimie comparée et de l'étude des altérations de la séquence polypeptidique pouvant être à l'origine de certains mécanismes adaptatifs. Dans le texte qui suit, nous décrirons la problématique qui a conduit aux premiers mutants artificiels de l' $\mathrm{Hb}$, pour aborder ensuite son application à la réalisation d'un transporteur d'oxygène.

\section{Hémoglobine recombinante et évolution}

Les chaînes de globine des vertébrés sont des protéines très anciennes dans l'échelle de temps de l'évolution biologique (plusieurs millions d'années) La molécule de globine monomérique ancestrale, de forte affinité pour l'oxygène, n'assurait qu'une fonction

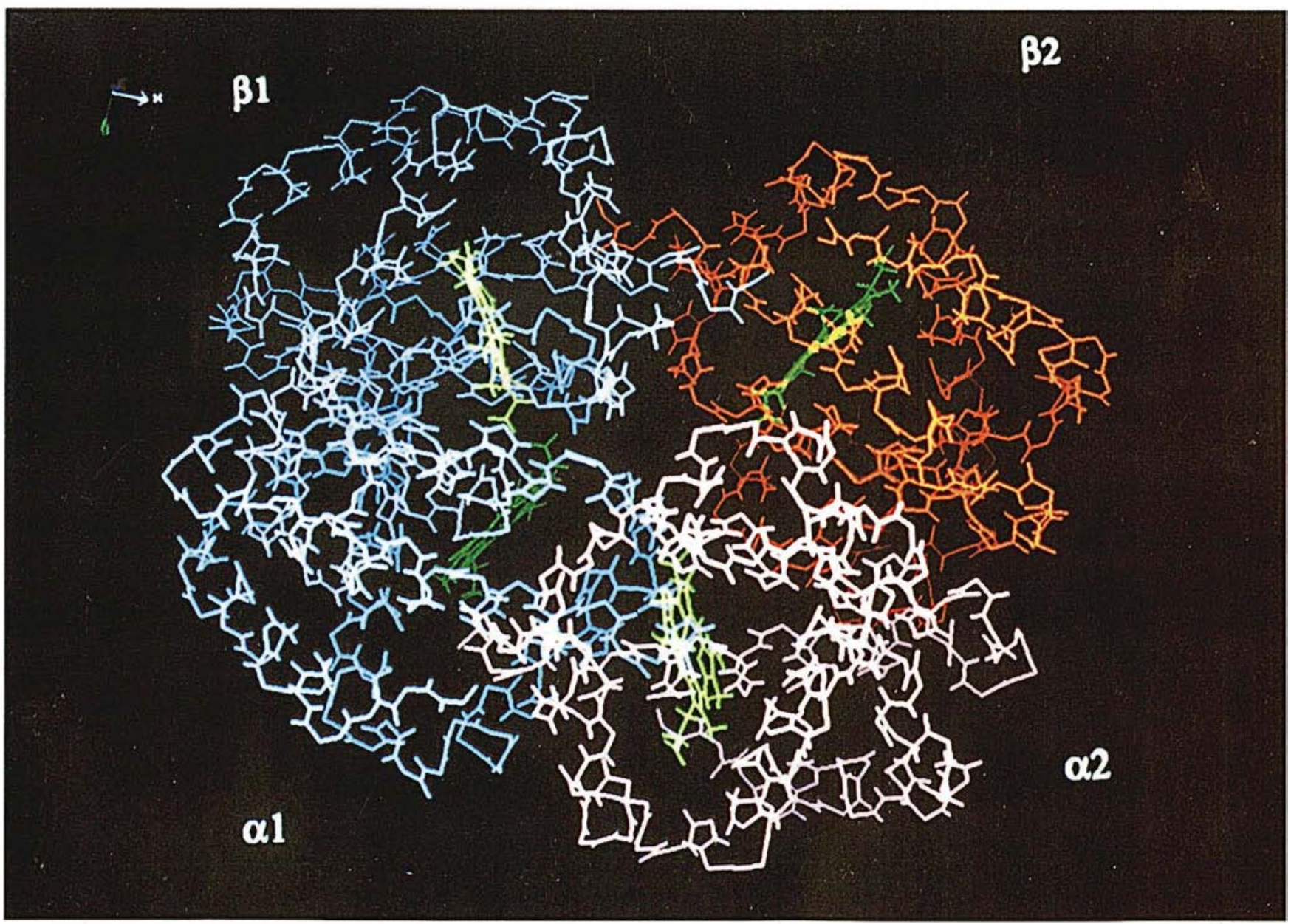

Figure 1. La molécule d'hémoglobine est un tétramère constitué de deux types de chaînes polypeptidiques $\alpha$ et $\beta$ (respectivement 141 et 146 acides aminés). Chaque sous-unité $\alpha$ est appariée avec une sous-unité $\beta$ pour former un dimère $\alpha \beta$ dont la stabilité est assurée par de nombreux contacts interchaînes. Cette zone de contact est rigide et quasi indépendante de l'état de saturation de l'hémoglobine par l'oxygène. Les deux dimères $\alpha \beta$ s'assemblent en tétramère, créant une zone de contact beaucoup plus lâche. La stabilité de cette interface, qui n'implique qu'un nombre limité de résidus, dépend de la concentration en hémoglobine et du pourcentage de saturation par l'oxygène des groupements héminiques (en vert). A faible concentration en hémoglobine et/ou à forte saturation en oxygène, l'interaction entre les deux dimères est affaiblie. Lors de l'oxygénation, les interfaces $\alpha_{1} \beta_{2}$ et $\alpha_{2} \beta_{1}$ sont le siège de modifications conformationnelles du tétramère par glissement-rotation entre dimères. Ces modifications sont responsables du fonctionnement allostérique, exprimé par la forme sigmoïde de la courbe de liaison de l'oxygène à I'hémoglobine. 
de stockage. L'apparition de deux types de chaînes de globine, $\alpha$ et $\beta$, leur assemblage en tétramère $\alpha_{2} \beta_{2}$ (figure 1, p. 791) avec l'acquisition de propriétés allostériques ont constitué une étape majeure de l'évolution de ces hémoprotéines. Assemblées en oligomères, les globines constituent une protéine "respiratoire" assurant le transport et la délivrance de l'oxygène aux tissus, pour des

\section{RÉFÉRENCES}

1. Geyer RF. Perfluorochemicals as oxygen transport vehicles. In : Chang TMS, Geyer RP, eds. Blood Substitutes. New York : Marcel Dekker, 1989 : 31-50.

2. Dellacherie E, Labrude P, Vigneron C, Riess JG. Synthetic carriers of oxygen. In : Bruck SD, ed. Critical Reviews in Therapeutic Drug Carriers Systems. Boca Raton, Florida : CRC Press, 1987 : 3-41.

3. Winslow RM. Hemoglobin-Based Red Cell Substitues. Baltimore - London : Johns Hopkins University Press, 1992.

4. Dickerson RE, Geis I. Hemoglobin : Structure, Function, Evolution, Pathology. Kenlo Park : Benjamin/Cummings Publishing Co, 1983.

5. Bunn HF, Forget BG. Hemoglobin : Molecular, Genetic and Clinical Aspects. Philadelphia: WB Saunders Co, 1985.

6. Perutz MF. Molecular anatomy, physiology, and pathology of hemoglobin. In : Stammatayanopoulos G, ed. Molecular Basis of Blood Diseases. Philadelphia : Saunders, 1987: 127-78.

7. IHIC Variants List. Hemoglobin 1991 ; $15: 139-217$

8. Fermi G, Perutz MF, Shaanan B, Fourme R. The crystal structure of human deoxyhaemoglobin at $1.74 \AA$ resolution. $J$ Mol Biol 1984 ; 175 : 159-74.

9. Nagai K, Thogersen HC. Generation of $\beta$-globin by sequence specific proteolysis of a hybrid protein produced in Escherichia coli. Nature 1984 ; 309 : 810-2.

10. Nagai K, Thogersen HC. Synthesis and sequence specific proteolysis of hybrid proteins produced in Escherichia coli. Meth Enzymol 1987 ; 153 : 461-81.

11. Tame J, Shih D, Pagnier J, Fermi G, Nagai K. Functional role of the distal valine (E11) residue of $\alpha$ subunits in human hae-

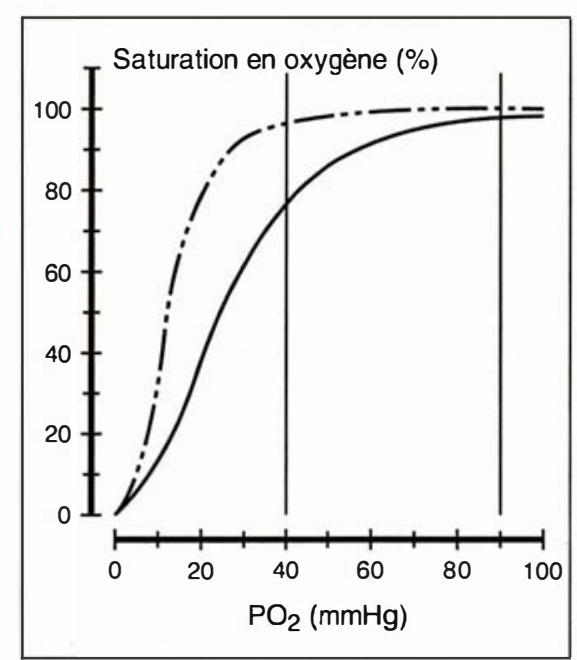

Figure 2. Courbes de liaison de l'oxygène à I'Hb obtenues avec une suspension de globules rouges humains avant $(-)$ et après (--) déplétion en DPG. La forme sigmoïde des courbes traduit la coopérativité de la liaison de l'oxygène à l'Hb. Les affinités, exprimées par la $p_{50} \quad\left(p \mathrm{O}_{2}\right.$ à $50 \%$ de saturation) sont respectivement de 25 et $10 \mathrm{~mm} \mathrm{Hg}$ avant et après déplétion en effecteur. L'intérêt physiologique du DPG est indiqué par les deux droites verticales, correspondant au niveau de $\mathrm{pO}_{2}$ du sang artériel et du sang veineux mêlés (respectivement $\mathrm{paO}_{2}=90$ et $\mathrm{p}_{\mathrm{v}} \mathrm{O}_{2}=$ $40 \mathrm{~mm} \mathrm{Hg}$. Entre ces limites, les différences de saturation en oxygène de I'Hb sont de $25 \%$ avant et de $5 \%$ après déplétion en DPG. Dans le globule rouge, la variation importante de l'affinité pour l'oxygène est due à l'existence d'une structure tétramérique et à la présence de l'effecteur $D P G$. La présence de DPG permet un transport normal de l'oxygène dans les conditions physiologiques de débit cardiaque et de concentration en $\mathrm{Hb}$ du sang. En absence de DPG, la forte affinité pour l'oxygène ne permet de remplir ce rôle qu'à la condition d'une forte augmentation du débit cardiaque et/ou de la concentration en Hb (polyglobulie). niveaux physiologiques de pressions partielles artérielle et veineuse en oxygène. Cet événement crucial a favorisé le développement des espèces vertébrées. Le caractère allostérique de la liaison de l'oxygène, traduit par la forme sigmoïde de la courbe d'oxygénation de l'Hb (figure 2), assure un fonctionnement extrêmement efficace de captation de l'oxygène dans les poumons ou les branchies et de sa délivrance aux tissus au niveau des capillaires. Malgré seulement $40 \%$ d'homologie entre les séquences des hémoglobines de vertébrés les plus éloignées, ces $\mathrm{Hb}$ présentent toutes la faculté de liaison coopérative et une affinité modérée pour l'oxygène (effets homotropes). La plupart des résidus impliqués dans cette fonction (poche de l'hème, interface $\alpha 1 \beta 2$ ), ont été conservés au cours de l'évolution. L'adaptation du transport de l'oxygène - selon les besoins métaboliques liés à la taille, à l'environnement, à la vie fotale... - est réalisée par des effets hétérotropes, c'està-dire par l'intervention de cofacteurs de l'hémoglobine qui, présents dans les globules rouges, modulent l'affinité pour l'oxygène : ions bicarbonates chez les crocodiles, ATP chez les amphibiens, inositol-pentaphosphate chez les oiseaux, 2,3-diphosphoglycérate (DPG) chez la plupart des mammifères (figure 2). En se liant de façon préférentielle à la désoxyhémoglobine (désoxyHb), ces effecteurs diminuent l'affinité pour l'oxygène. Les mécanismes adaptatifs correspondent à des modifications soit des sites de liaison de ces effecteurs, soit de leur concentration cellulaire. Les structures responsables des effets homotropes sont, en revanche, remarquablement stables.

C'est à propos d'une question liée à la physiologie comparée et à l'évolution des espèces que commence l'histoire des $\mathrm{Hb}$ recombinantes. Afin de remonter pas à pas l'évolution, et de rechercher les résidus responsables des fonctions spécifiques d'une espèce, des chercheurs de Cambridge (Grande-Bretagne) ont utilisé les possibilités offertes par le développement des techniques de biologie moléculaire. En 1984, Nagai et Thogersen mettent au point un système d'expression des sous-unités de globine humaine, $\beta$ puis $\alpha$, dans Esche- 
richia coli [9-11]. Les sous-unités sont synthétisées sous la forme d'une protéine de fusion. Après extraction et purification de cette protéine, les chaînes de globine sont libérées par un clivage spécifique (facteur de coagulation $\mathrm{X}$ activé). L'Hb reconstituée à partir de ces sous-unités non modifiées dans leur séquence, présente des propriétés fonctionnelles similaires à celles d'une $\mathrm{Hb}$ humaine native (Tableau I). L'utilisation de ce système d'expression, couplée à celle de la mutagenèse dirigée, autorisait alors la production de mutants artificiels.

\section{L'exemple de l'effet Root}

L'affinité pour l'oxygène des hémoglobines de la plupart des espèces de vertébrés diminue à $\mathrm{pH}$ acide. Cet effet hétérotrope, dû à la liaison préférentielle des ions $\mathrm{H}^{+}$à la désoxyhémoglobine est connu sous le nom d'effet Bohr. Il assure le transport des ions $\mathrm{H}^{+}$et du gaz carbonique produits par le métabolisme cellulaire, et le maintien du $\mathrm{pH}$ plasmatique dans d'étroites limites. Dans certaines hémoglobines de poissons téléostéens, l'effet Bohr est considérablement accru en dessous de $\mathrm{pH} 7$ et constitue l'effet Root. Les hémoglobines à effet Root lient l'oxygène avec une si faible affinité à $\mathrm{pH}$ acide, qu'elles demeurent partiellement désoxygénées, même sous oxygène pur. La comparaison des séquences d'acides aminés des châ̂nes de $\beta$-globine de diverses espèces et de celles des hémoglobines à effet Root conduisit Perutz et Brunori à identifier une substitution spécifique de l'Hb de ces espèces : le remplacement du résidu $\beta 93$ Cys (présent dans les $\mathrm{Hb}$ de vertébrés) par un résidu Ser [4]. L'hypothèse de Perutz et Brunori, attribuant à cette seule substitution l'origine de l'effet Root [12], a été testée en introduisant par mutagenèse dirigée un résidu Ser en position 93 des chaînes $\beta$ de l'hémoglobine humaine. Seule ou associée à d'autres modifications de la chaîne $\beta$, la substitution $\beta 93$ Cys $\rightarrow$ Ser dans l'Hb humaine ne permet pas de reproduire l'effet Root $[13,14]$. Ces résultats ont souligné la complexité des multiples interactions électrosta-

Tableau I

PROPRIÉTÉS FONCTIONNNELLES D'HÉMOGLOBINES RECOMBINANTES

\begin{tabular}{|llccc|}
\hline Hb & & $\begin{array}{c}\mathbf{P}_{\mathbf{5 0}} \\
\mathbf{m m}^{\text {H }} \mathbf{H g}\end{array}$ & $\mathbf{n}_{\mathbf{5 0}}$ & effet DPG \\
\hline HbA & native & 5 & 2,8 & 0,5 \\
& recombinante & 4,5 & 2,4 & 0,5 \\
Hb Knossos & native & 7,7 & 2,7 & 0,38 \\
$(\beta 27$ Ala $\rightarrow$ Ser) & recombinante & 6,4 & 2,5 & 0,48 \\
\hline
\end{tabular}

Les sous-unités $\beta$ recombinantes ont été exprimées sous forme de protéine de fusion dans E. coli. Après purification, les chaînes $\beta$ ont été libérées par clivage spécifique, et associées à des sous-unités $\alpha$ natives en présence d'hème. $P_{50}$ et $n_{50}$ : pression partielle en oxygène et index de coopérativité à demi-saturation; effet DPG: $\triangle \log P_{50}$ $+1-1 \mathrm{mM} D P G$ à $p H$ 7,2. Conditions expérimentales : tampon Bis-Tris $50 \mathrm{mM} p H 7,2$; $\mathrm{NaCl} 100 \mathrm{mM}$; EDTA $50 \mu \mathrm{M}$; catalase $20 \mu \mathrm{g} / \mathrm{ml} ; 25^{\circ} \mathrm{C}$.

tiques dans l'hémoglobine et de leur participation à l'expression des propriétés fonctionnelles. Ces données expérimentales doivent être prises en compte dans toute recherche fondamentale ou appliquée.

Hémoglobine recombinante et transporteur d'oxygène artificiel

Un autre champ d'application de l'expression d'Hb recombinante s'imposait rapidement : synthétiser une $\mathrm{Hb}$ susceptible d'être utilisée comme transporteur d'oxygène in vivo.

L'idée d'utiliser des solutions d'Hb à des fins transfusionnelles, à la fois logique et ambitieuse, n'est certes pas nouvelle [2, 3]. L'utilisation de solutions d' $\mathrm{Hb}$, humaine ou bovine, même débarrassées des débris membranaires néphrotoxiques, n'a toutefois guère dépassé le stade de l'expérimentation sur l'animal. Les problèmes sont en effet multiples (Tableau II).
- La concentration de l'Hb dans les globules rouges est de $300 \mathrm{~g} / \mathrm{l}$ soit $5 \mathrm{mM}$. Il est impossible d'injecter des solutions aussi concentrées en $\mathrm{Hb}$. Leur viscosité augmenterait considérablement le travail cardiaque. En exerçant une pression oncotique très élevée, ces solutions seraient responsables de déshydratation cellulaire.

- Hors du confinement dans les globules rouges, l'affinité pour l'oxygène de l'Hb en solution n'est plus modulée par le DPG (qui n'existe pas dans le plasma). En l'absence de ce cofacteur, l'Hb humaine en solution fixe l'oxygène avec une affinité trop élevée pour délivrer l'oxygène aux tissus (figure 2).

- Enfin, en solution diluée, la structure tétramérique de l'Hb n'est pas préservée : la molécule se dissocie en dimères $\alpha \beta$. Ces dimères sont néphrotoxiques et rapidement filtrés par le rein, ce qui limite la

\section{Tableau ॥}

\section{SOLUTION D'HB ET TRANSPORTEUR D'OXYGĖNE ARTIFICIEL : LE CAHIER DES CHARGES}

- Élimination des risques infectieux et immunologiques

- Diminution de l'affinité pour l'oxygène (sang normal : $27 \mathrm{~mm} \mathrm{Hg}$ à $37^{\circ} \mathrm{C}$ )

- Stabilisation de la structure tétramérique

- Augmentation de la concentration sans augmentation de la pression oncotique

- Diminution de la vitesse de formation de metHb

- Stabilité et disponibilité

- Pas de limites dans l'approvisionnement

- Coût raisonnable 


\section{RÉFÉRENCES}

12. Perutz MF, Brunori M. Stereochemistry of cooperative effects in fish and amphibian haemoglobins. Nature 1982 ; 299 : 421-6.

13. Nagai K, Perutz MF, Poyart C. Oxygen binding properties of human mutan hemoglobins synthesized in Escherichia coli. Proc Natl Acad Sci USA 1985; 82 : 7252-5

14. Luisi B, Nagai K. Cyrstallographic analysis of mutant human haemoglobins made in Escherichia coli. Nature 1986; 320 : 555-6.

15. Zhang L, Levy A, Rifkind JM Autooxidation of hemoglobin enhanced by dissociation into dimers. J Biol Chem 1991 ; 266 : 24698-701

16. Hoffman SJ, Looker DL, Roehrich JM, et al. Expression of fully functional tetrameric human hemoglobin in Escherichia coli. Proc Natl Acad Sci USA 1990 ; 87 : 8521-5.

17. Wagenbach M, O'Rourke K, Vitez L, et al. Synthesis of wild type and mutant human hemoglobins in Saccharomyces cerevisiae. Biotechnology 1991; 9 : 57-61.

18. Huang $\mathrm{Y}$, Pagnier J, Magne $\mathrm{P}$, et al. Structure and function of hemoglobin variants at an internal hydrophobic site consequences of mutations at the $\beta 27$ (B9) position. Biochemistry 1990 ; 29 : 7020-3.

19. Pagnier J, Baudin-Chich V, Lacaze $\mathrm{N}$, Bohn B, Poyart C. Haemoglobin $\alpha_{2} \beta_{2} 23$ $\mathrm{Val} \rightarrow$ Ile produced in Escherichia coli facilitate $\mathrm{Hb} \mathrm{S}$ polymerization. Br J Haematol 1990 ; $74: 532-4$

20. Looker D, Abbott-Brown D, Cozart P, et al. A human recombinant haemoglobin designed for use as a blood substitute. Nature 1992 ; 356 : 258-60.

21. Moo-Penn WF, Wolff JA, Simon G Vacek M, Jue DL, Johnson MH. Hemoglobin Presbyterian : $\beta 108$ (G10) Asparagine $\rightarrow$ Lysine. A hemoglobin variant with low oxygen affinity. FEBS Lett 1978 ; 92 : 53-6.

22. Behringer RR, Ryan TM, Reilly MP, et al. Synthesis of functional human hemoglobin in transgenic mice. Science 1989 ; 245 : 971-3.

Depuis la rédaction de cet article, a été publié dans Biotechnology:

Swanson ME, Martin MJ, O'Donnel JK, et al. Production of functional human hemoglobin in transgenic swine. Biotechnology 1992 ; 10 : 557-9. durée de vie de l'Hb dans l'organisme. Ils sont en outre plus sensibles à l'oxydation que l' $\mathrm{Hb}$ tétramérique [15].

Diverses modifications biochimiques de l'Hb ont été réalisées pour pallier ces limitations. Ces travaux sont décrits de façon exhaustive dans d'excellentes revues parmi lesquelles celles de Dellacherie et al. [2] et de Winslow [3]. Il reste que le problème majeur posé par cette stratégie réside dans l'utilisation d'Hb provenant de sang humain : le risque de contamination virale n'est pas éliminé. L'approvisionnement en sang humain périmé constitue une autre limite, et ne saurait suffire aux besoins. Certains auteurs ont envisagé l'utilisation d'Hb bovine, disponible en grande quantité pour un faible coût. Cette Hb présente l'avantage d'une affinité intrinsèque pour l'oxygène, réduite en absence de DPG. Cependant l'absence de risques immunologiques, même faibles, reste à établir.

Les mutants naturels et artificiels de l'Hb ont apporté la preuve que la modification d'un seul résidu peut altérer, dans certains cas de façon spectaculaire, les propriétés de la molécule ( $\mathrm{Hb} \mathrm{S}, \mathrm{Hb}$ instables...). L'expression de sous-unités de globine, modifiées ou non, est possible dans $E$. Coli $[9,10,16]$. Elle a également été mise au point dans une levure, Saccharomyces cerevisiae [17]. Peut-on alors, avec quelques mutations bien choisies, réaliser un produit réunissant les propriétés exigées : faible affinité pour l'oxygène, stabilité tétramérique, limitation de l'autooxydation des hèmes dans les solutions d'Hb (Tableau II) ? En termes d'affinité pour l'oxygène et de stabilité tétramérique, la réponse est affirmative.

\section{Les systèmes d'expression}

Les premiers systèmes d'expression développés pour produire de l'Hb recombinante permettent la production séparée des sous-unités $\alpha$ et $\beta$ dans E. Coli, sous la forme de protéines de fusion insolubles. Les procédures de purification de la protéine, puis de reconstitution du tétramère d'Hb, sont lourdes [9-11]. Ces méthodes, difficilement transposables à l'échelle industrielle, restent toute- fois extrêmement utiles en recherche. En effet, la protéine de fusion représente 15 à $20 \%$ des protéines totales synthétisées par la bactérie, rendement qui assure l'obtention de quantités d'Hb suffisantes pour les études fonctionnelles et structurales. Par ailleurs, l'identité structurale et fonctionnelle des tétramères natif et recombinant sauvages autorise la comparaison directe des propriétés d'Hb recombinantes mutées avec celles d'une $\mathrm{Hb}$ normale native.

Hoffman $e t$ al. ont récemment mis au point un système de coexpression des sous-unités $\alpha$ et $\beta$ dans $E$. coli. Utilisant l'hème endogène, les $\alpha$ - et $\beta$ globines s'assemblent en $\mathrm{Hb} \quad \alpha_{2} \beta_{2}$ ayant acquis sa structure quaternaire, et qui s'accumule sous forme soluble dans la bactérie. Cette Hb fixe l'oxygène de façon réversible et coopérative, mais ne présente pas toutes les propriétés fonctionnelles d'une $\mathrm{Hb}$ humaine normale [16]. Les anomalies résultent de la persistance du résidu méthionine en position $\mathrm{N}$-terminale des sous-unités, résidu mal clivé par le système enzymatique des procaryotes. Pour pallier cet inconvénient, ces mêmes auteurs ont réalisé la coexpression des sous-unités $\alpha$ et $\beta$ dans $S$. cerevisiae. L'Hb obtenue est soluble; sa structure et ses propriétés fonctionnelles sont identiques à celles d'une $\mathrm{Hb}$ humaine native [17]. Les rendements d'expression dans les levures sont malheureusement faibles, 3 à $5 \%$ des protéines totales, ce qui limite, au moins actuellement, leur utilisation dans le cadre d'un projet de développement industriel.

\section{Diminution de l'affinité pour l'oxygène}

Des mutations naturelles ont été reproduites par mutagenèse dirigée : $\beta 27 \mathrm{Ala} \rightarrow$ Ser, mutation de l'Hb Knossos ; $\beta 23 \mathrm{Val} \rightarrow$ Ile, mutation associée à la mutation drépanocytaire dans l'Hb S-Antilles; $\beta 108$ Asn $\rightarrow$ Lys, mutation de l'Hb Presbyterian. Ces mutations naturelles sont connues pour entraîner une diminution de l'affinité de l' $\mathrm{Hb}$ pour l'oxygène [7]. Comme les $\mathrm{Hb}$ natives, les $\mathrm{Hb}$ recombinantes réalisées d'après ces modèles présentent une diminution d'affinité. Les effets hétérotropes, effet Bohr, liaison des chlorures et des organophosphates, 


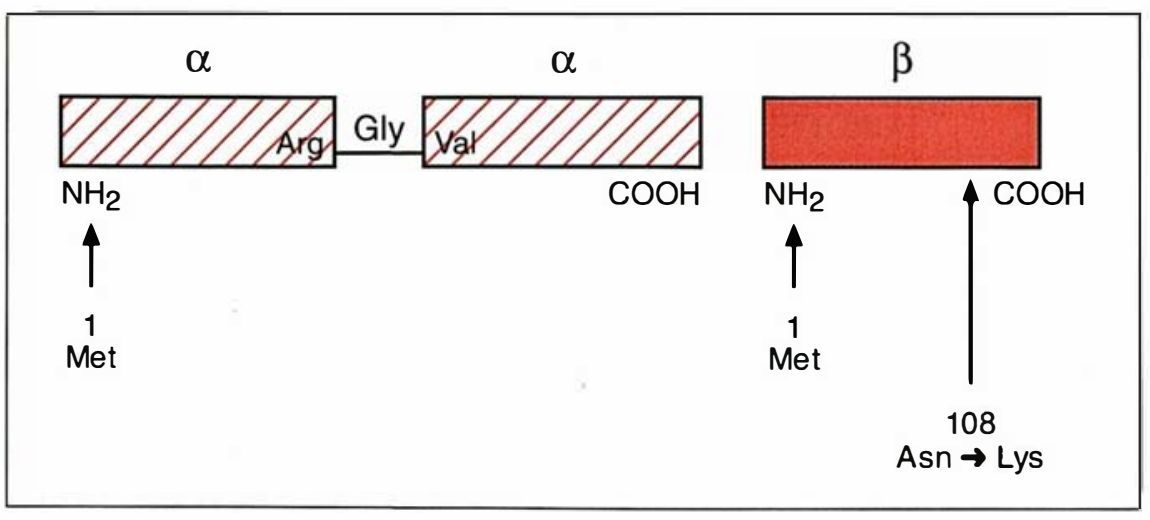

Figure 3. Représentation schématique des sous-unités $\alpha$ et $\beta$ synthétisées dans E. coli par Looker et al. [20]. Les sous-unités sont synthétisées sous le contrôle d'un même promoteur (promoteur TAC). Les chaînes $\alpha$ sont associées en dimères par l'intermédiaire d'un résidu glycyl. Le résidu Asn 108 de la chaîne $\beta$ a été remplacé par une Lys. Cette mutation est connue pour entraîner une diminution de l'affinité pour l'oxygène (Hb Presbyterian, 21). Les valines terminales ont été remplacées par des méthionines. Un dimère di- $\alpha$ et deux sous-unités $\beta$ s'assemblent en fixant l'hème endogène pour former un tétramère d'Hb stable, d'affinité pour l'oxygène comparable à celle du sang total. (D'après [16] et [20].)

sont similaires à ceux observés avec les $\mathrm{Hb}$ mutées natives (Tableau I) [17-19].

Autres exemples : le remplacement de la phénylalanine $\beta 41$ par une tyrosine, et celui de l'histidine $\beta 2$ par une méthionine (sur le modèle de l' $\mathrm{Hb}$ bovine) entraînent également une diminution de l'affinité pour l'oxygène $(\mathrm{J}$. Pagnier et al., résultats non publiés). Ces résultats démontrent la possibilité d'obtenir une molécule d'Hb recombinante capable, en l'absence de DPG, de remplir les conditions d'affinité d'un transporteur d'oxygène efficace. L'association de certaines de ces mutations permettrait éventuellement de moduler l'affinité en fonction des indications de la transfusion.

$\mathrm{Hb}$ recombinante et stabilisation de la structure tétramérique

Une étape importante a été ensuite franchie par Looker et al. [20] qui ont modifié la construction plasmidique, permettant la coexpression des sousunités $\alpha$ et $\beta$ dans $E$. coli : les sousunités $\alpha$ sont produites sous forme dimérique, $\alpha-\alpha$, liées par l'intermédiaire d'un résidu glycyl entre l'arginine terminale d'une sous-unité et la valine initiale de l'autre. Sous cette forme, les sous-unités $\alpha$ s'assemblent correctement aux sous-unités partenaires $\beta$ et à l'hème pour former une $m / s n^{\circ} 8$, vol. 8, octobre 92 modification de résidus de la partie distale de la poche de l'hème.

\section{Les animaux transgéniques}

A la différence des autres protéines recombinantes utilisées en thérapeutique humaine, les quantités d'Hb qui doivent être produites à partir des cellules procaryotes ou eucaryotes sont telles que les problèmes de développement industriel sont importants. Une solution pourrait venir de la création d'animaux transgéniques. Depuis 1989, on sait introduire les séquences codantes des gènes de l'Hb humaine flanquées de leur séquences promotrices dans le génome de souris [22]. Ces souris transgéniques synthétisent de l'Hb A humaine (5\% environ de l'Hb totale). Plus récemment, la presse quotidienne* a rapporté l'obtention, par des chercheurs américains, de porcs transgéniques qui synthétisent 10 à $15 \%$ de leur $\mathrm{Hb}$ totale sous forme d'Hb humaine.

(étraméture tétra structu est ainsi stabilisée par une liaison covalente entre les dimères $\alpha \beta$ (figure 3).

Les auteurs ont comparé, chez le rat, les taux de rétention intravasculaire des solutions d'Hb avec et sans lien peptidique. Bien qu'augmentée d'un facteur 3 à 4, la demi-vie de l'Hb recombinante "stabilisée " est de l'ordre de deux heures. Pour augmenter encore la demi-vie intravasculaire, on peut envisager la synthèse de di- ou tri-tétramères par l'introduction d'un résidu cystéyl à la surface des chaînes $\alpha$ ou $\beta$, dans le but de créer des ponts disulfures. Ce type de construction permettrait en outre d'augmenter la concentration en $\mathrm{Hb}$ des solutions injectables, sans modifier la pression oncotique. La tolérance rénale à l'administration du tétramère stabilisé a également été vérifiée. Chez le chien, la fonction rénale n'est pas affectée après remplacement de $30 \%$ du volume sanguin par cette solution d'Hb recombinante.

\section{Oxydabilité}

L'Hb en solution est rapidement oxydée en metHb, forme impropre au transport de l'oxygène. La stabilisation de la structure tétramérique limite la vitesse d'oxydation, qui pourrait encore être ralentie par

\section{Conclusion}

Depuis la synthèse de la première sous-unité $\beta$ de globine en 1984 [9], les avancées ont été fantastiques: réalisation d'une $\mathrm{Hb}$ de faible affinité pour l'oxygène et dont la structure tétramérique est stable en solution. L'ingénierie de l'Hb, avec la possibilité de modifier les séquences codantes des chaînes de globine, permettrait de rendre disponible un "produit fini " ne nécessitant plus de traitement ultérieur autre que la purification. Actuellement, la réalisation d'un produit de première génération - soluté macromoléculaire de réanimation utilisable en urgence, apportant 10 à 20 fois plus d'oxygène qu'une solution d'albumine - ou d'une molécule de synthèse, dépourvu de risques infectieux et immunologiques, paraît un objectif raisonnable, réalisable à moyen terme. La fabrication de "globules rouges " artificiels, c'est-à-dire l'encapsulation de l'Hb recombinante dans un vecteur biologique compatible est, en revanche, un projet à plus long terme compte tenu des nombreux problèmes qui restent à régler

* Le Monde daté du 18-6-1991.

(Summary p. suivante) 


\section{Summary}

Recombinant hemoglobin and blood substitute

The search for safe alternatives to conventional blood transfusion has focused on the use of synthetic perfluorochemicals and stroma-free $\mathrm{Hb}$ solutions purified from outdated bank blood. The use of human blood does not eliminate infectious risks. An alternative for a novel source of $\mathrm{Hb}$ are microorganisms whose genome has been modified to contain globin genes. Several problems must be solved before considering the clinical use and the industrial development of recombinant $\mathrm{Hb}$ solutions : without 2,3-DPG, the oxygen affinity of these solutions is so high that very little oxygen is unloaded to the tissues ; the dissociation into dimers results in short intravascular half-life ; the rate of autooxidation is high. Expression of $\alpha$ and $\beta$-globins in Escherichia coli enables one to introduce appropriate mutation(s) in the $\mathrm{Hb}$ molecule resulting in a synthetic $\mathrm{Hb}$ with reduced oxygen affinity and normal allosteric properties, allowing in vivo oxygen binding and delivery. Functional tetrameric $\mathrm{Hb}$ has been recently produced in a soluble form either in E. coli or in yeast. The expression of dimeric $\alpha-\alpha$ subunits linked by peptide bonds allows the formation of stabilized $\mathrm{Hb}$ tetramers. Further studies should be aimed at limiting the oxidation of the heme groups in the $\mathrm{Hb}$ solutions, at the development of highly safe purification techniques, and at scaling-up procedures. 